\title{
Deutsch-Französische Partnerschaften
}

von Hannsjörg Kowark

Zwischen den Städten Stuttgart und Straßburg bestehen im Rahmen einer Städtepartnerschaft seit fast 50 Jahren enge und freundschaftliche Beziehungen. Im Mittelpunkt der Partnerschaft stand von Anfang an die Aussöhnung zwischen Frankreich und Deutschland.

Als 2003 das Goethe-Institut in Paris anlässlich des 40. Jahrestages des ElyséeVertrages Partnerschaften zwischen den Bibliotheken angeregt hat, um durch grenzübergreifende Aktivitäten im Kultur- und Bildungsbereich aktiv zur Förderung der europäischen Integration beizutragen, hat die Württembergische Landesbibliothek diese Anregung bereitwillig aufgegriffen. Im Sommer 2003 wurden erste Kontakte mit der Bibliothèque Nationale et Universitaire (BNU) in Straßburg aufgenommen. Regelmäßige Treffen zwischen den Direktoren beider Bibliotheken sowie Praktika einzelner Kolleginnen und Kollegen in der Partnerbibliothek folgten. Bereits ein Jahr später konnte im Oktober 2004 die Ausstellung der BNU „Impressions d'Europe - Trésors de la BNUS entre France et Allemagne" in der Württembergischen Landesbibliothek eröffnet werden.

Als erstes großes Gemeinschaftsprojekt haben die BNU und die WLB seit 2005 die Ausstellung „In Papiergewittern - Orages de papier“ vorbereitet. Die Ausstellung zeigt in beeindruckender Weise den Ersten Weltkrieg als den ersten umfassenden Medienkrieg des 20. Jahrhunderts, der eine beispiellose Medienflut - ein regelrechtes Papiergewitter - in Form von Plakaten, Flugblättern, Schützengraben-Zeitungen, Tagebüchern, Filmen und Fotos ausgelöst hat. Bibliotheken und private Sammler haben die Spuren dieses Medienkrieges gesichert, so dass die Kriegssammlungen des Ersten Weltkrieges für die Forschung eine unschätzbare Quelle darstellen.

Vor 95 Jahren lagen sich unsere Großväter auf der deutschen und französischen Seite noch in den Schützengräben gegenüber, so dass das Ausstellungsprojekt auch als Zeichen der großen Freundschaft zwischen unseren beiden Ländern zu werten ist, die die schmerzhaften Erfahrungen der Vergangenheit endgültig überwunden haben.

Es war deshalb für beide Bibliotheken eine besonders große Freude, dieses Partnerschaftsabkommen im Rahmen der Eröffnung der Ausstellung „In Papiergewittern“ am 12. November 2008 in Straßburg unterzeichnen zu können.

Die Ausstellung, die unter der Schirmherrschaft von Herrn Minister Peter Frankenberg steht, wurde am 12. März 2009 in der WLB von Herrn Staatssekretär Dr. Dietrich Birk eröffnet und war bis zum 25. April 2009 in der Landesbibliothek zu sehen. Als weiterer Ausstellungsort ist für 2010 Paris geplant.

Die von Konrad Adenauer und Charles de Gaulle 1963 formulierten Ziele finden mit dieser Ausstellung und der Partnerschaft zwischen Württembergischer Landesbibliothek und Bibliothèque Nationale et Universitaire erneut eine Bestätigung. 


\section{Die deutsch-französische Zusammenarbeit Kernpunkt der europäischen Einigung} von Vera Trost

Innerhalb der Zusammenarbeit mit den 27 EU-Mitgliedsstaaten nimmt die deutschfranzösische Partnerschaft durch ihre Geschichte, ihre Intensität und ihre besondere institutionelle Ausgestaltung eine herausgehobene Stellung ein. Die deutschfranzösische Aussöhnung und der Aufbau einer Partnerschaft waren unmittelbar nach dem zweiten Weltkrieg Kern und Ausgangspunkt des europäischen Einigungsprozesses.

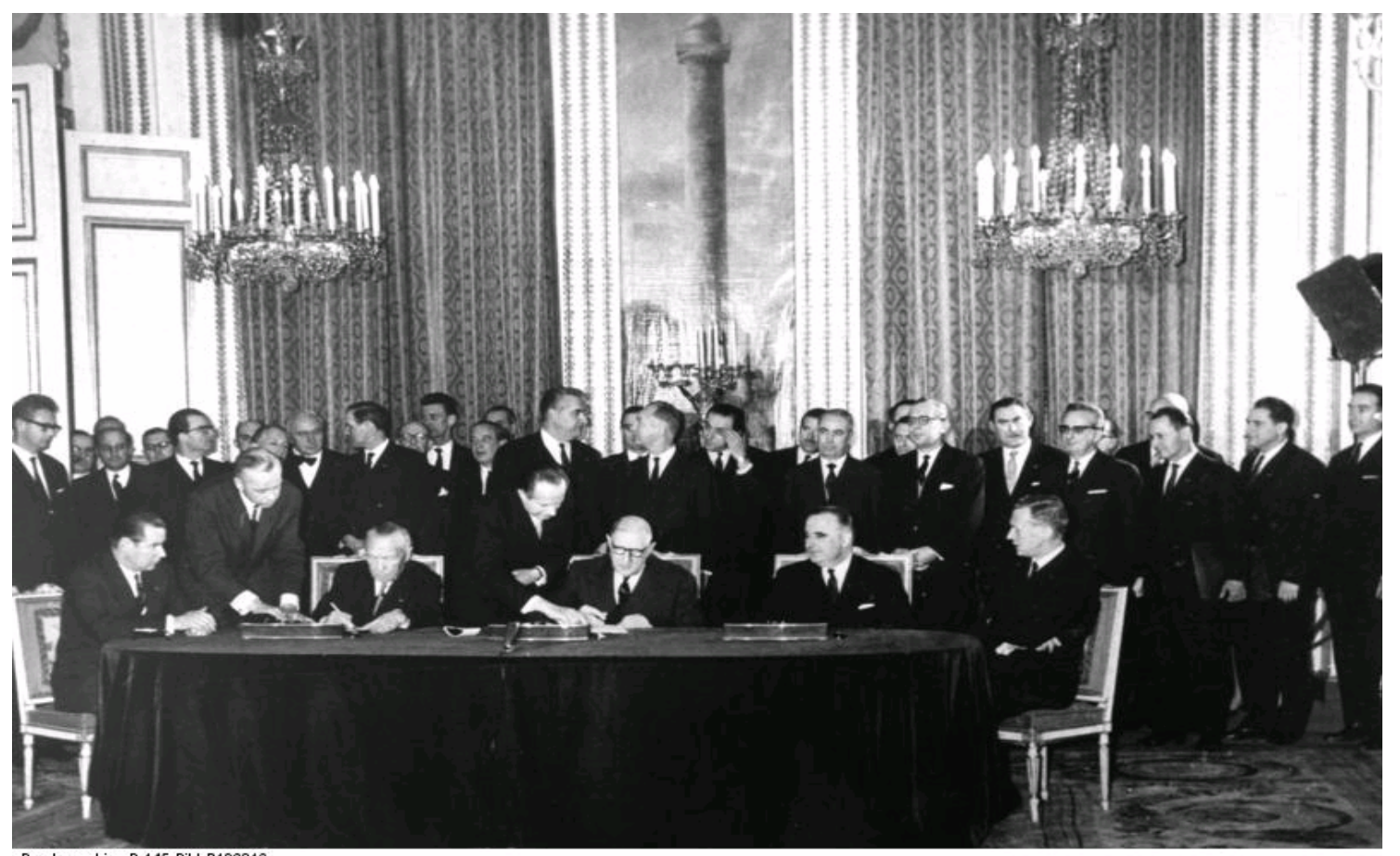

Bundesarchiv, B 145 Bild-P106816
Foto: 0.Ang. | 22. Januar 1963

Bundeskanzler Konrad Adenauer und der französische Staatspräsident Charles de Gaulle unterzeichnen den als Élysée-Vertrag bezeichneten deutsch-französischen Freundschaftsvertrag.

Élysée-Palast Paris, 22. Januar 1963

Dieses Abkommen über die deutsch-französische Zusammenarbeit hat die beiden Nachbarn in Europa nach langer „Erbfeindschaft“ und verlustreichen Kriegen seitdem immer mehr zusammengeführt. Der Vertrag verpflichtet beide Regierungen zu Konsultationen in allen wichtigen Fragen der Außen-, Sicherheits-, Jugend- und Kulturpolitik. Ebenso wurden regelmäßige Treffen auf Regierungsebene beschlossen, die in seither regelmäßigen Abständen durchgeführt wurden. 


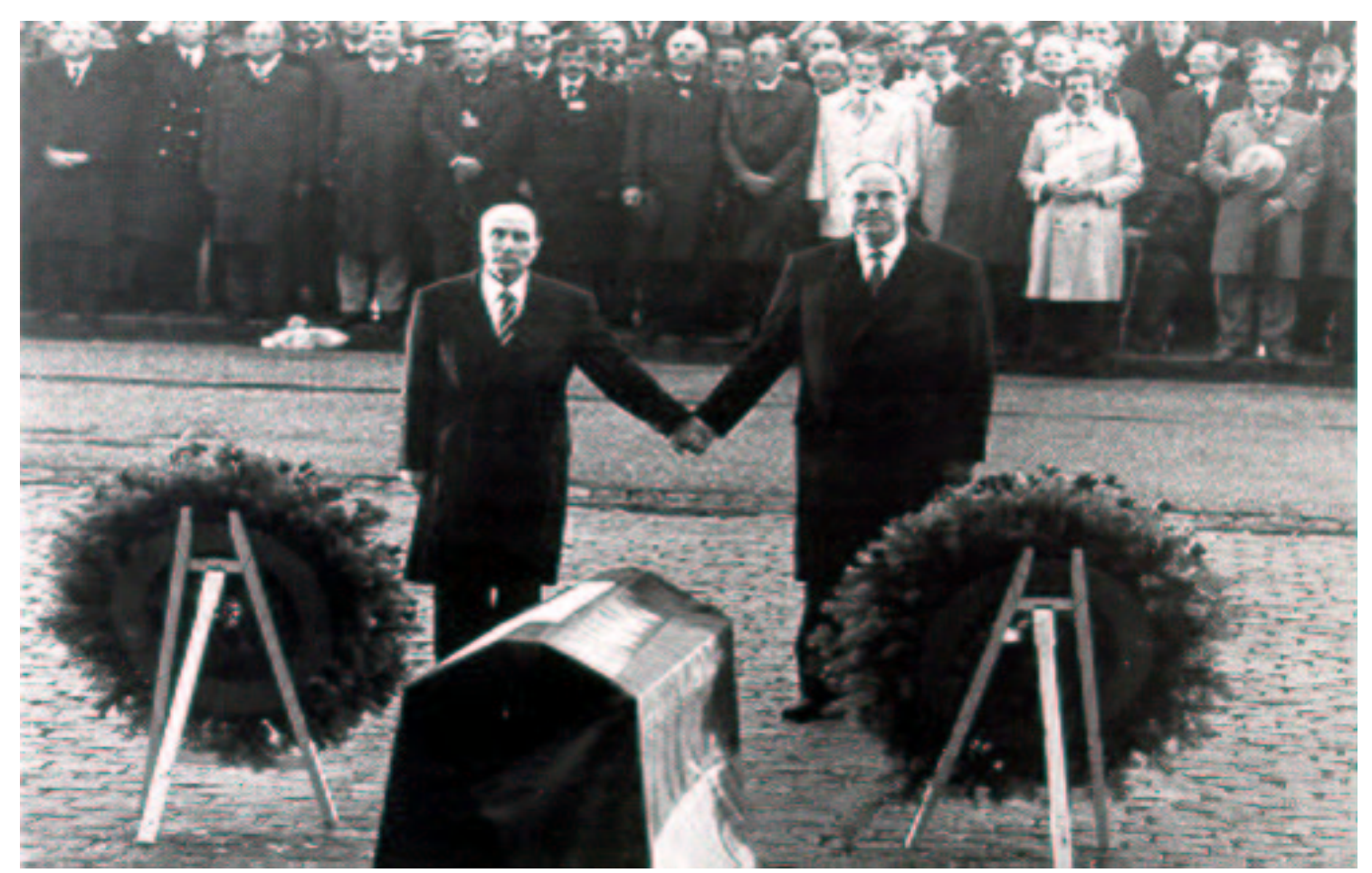

Der französische Staatspräsident François Mitterrand und Bundeskanzler Helmut Kohl gedenken in Verdun gemeinsam der Toten beider Weltkriege

Verdun, 22. September 1984

Fotografie: Bundesbildstelle, Bonn

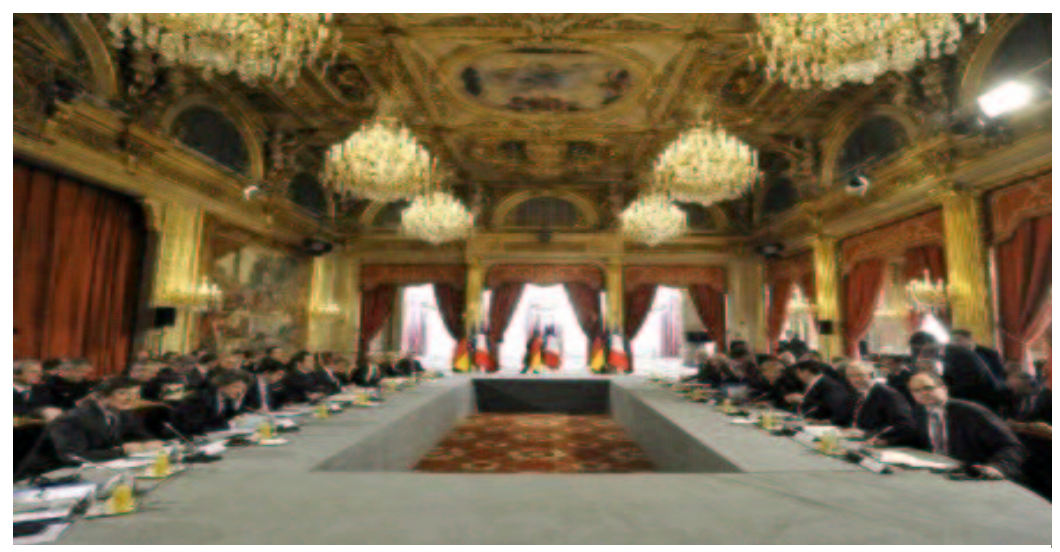

Der 10. Deutsch-Französische Ministerrat findet in Paris statt. Der Ministerrat, die gemeinsame Sitzung der beiden Kabinette, wird in dieser Form seit 2003 jeweils im Frühjahr und im Herbst abwechselnd in Frankreich und in Deutschland abgehalten. 


\section{Die Städtepartnerschaft zwischen Stuttgart und Straßburg}

von Vera Trost

Noch vor dem Besuch des französischen Staatspräsidenten Charles de Gaulle in Baden-Württemberg und Stuttgart im September 1963 und dem Abschluss des deutschfranzösischen Vertrages am 22. Januar 1963 hatten der Straßburger Oberbürgermeister Pierre Pflimlin und Stuttgarts Oberbürgermeister Dr. Arnulf Klett am 26. Mai 1962 im Stuttgarter Rathaus die Partnerschaftsurkunden unterzeichnet: Neben dem Aufbau besonderer freundschaftlicher Beziehungen zwischen den Bürgern beider Städte standen Friedenssicherung und die deutsch-französische Versöhnung im Vordergrund.

Nur 110 Kilometer Luftlinie trennen Stuttgart und Straßburg, die Reise mit dem französischen Schnellzug TGV dauert inzwischen nur eine Stunde und 16 Minuten, mit dem Auto sind es etwa zwei Stunden: beste Voraussetzungen für eine lebendige Beziehung zwischen den beiden Städten: der Reiz des Spektakulären ist längst der Normalität des Vertrauten gewichen.

Die Städtepartnerschaft war von Anfang an sehr rege, hat sich im Laufe ihres bald 50 jährigen Bestehens zu einem lebendigen Mosaik aus vielfältigen Begegnungen von Bürgern und Institutionen beider Städte in allen gesellschaftlichen Bereichen entwickelt und ist heute im Herzen der Menschen verankert.

Der deutsch-französische Motor in Straßburg und Stuttgart stockt also nicht, wie viele behaupten, sondern ist moderner und leiser geworden, bei höherer Leistungsfähigkeit und Flexibilität.

Stuttgart und Straßburg haben 2002 zum 40-jährigen Jubiläum nochmals ihre Zusammenarbeit mittels einer Urkunde bekräftigt, in der sie unterstreichen, dass sie weiterhin die Begegnungen ihrer Bürgerinnen und Bürger unterstützen und projekt- und themenbezogen zusammenarbeiten wollen sowie den internationalen Fachaustausch forcieren und die kommunale Entwicklung auf europäischer und internationaler Ebene gemeinsam vorantreiben möchten.

Beispiele für den regen, oft langjährigen Austausch oder einmalige Begegnungen gibt es viele: das Straßenrennen Stuttgart - Straßburg, regelmäßige Schwimmmeisterschaften, Schüleraustausche, europäische und internationale Jugendprojekte, Sportprojekte, Chorbegegnungen, Frauentreffs, Kunstausstellungen und viele Veranstaltungen im Rahmen der alljährlich stattfindenden Französischen Woche, an der sich die Württembergische Landesbibliothek von Anfang an beteiligte. 


\section{Die Bibliothekspartnerschaft zwischen der Bibliothèque Nationale et Universitaire Strasbourg und der Württembergischen Landesbibliothek Stuttgart}

von Vera Trost

Anlässlich des 40. Jahrestages der Unterzeichnung des "Elysée-Vertrags" regte das Goethe-Institut in Paris an, dass sich deutsche und französische Bibliotheken partnerschaftlich verbinden sollten. Ziel ist es, anhand von Partnerschaften zwischen deutschen und französischen Bibliotheken ein aktives Netz von Informationsknotenpunkten aufzubauen. Diesem Vorschlag folgten die Württembergische Landesbibliothek Stuttgart und die BNU Strasbourg. Nach fünf Jahren aktiver und erfolgreicher Zusammenarbeit haben beide Bibliotheken ihre Partnerschaft offiziell besiegelt.

Die feierliche Unterzeichnung erfolgte in Strasbourg am 12. November 2008 in Anwesenheit von

- Albert Poirot, Administrateur de la Bibliothèque Nationale et Universitaire de Strasbourg

- Dr. Hannsjörg Kowark, Direktor der Württembergischen Landesbibliothek

- Claire Lovisi, Recteur de l'Académie de Strasbourg, Chancellier des Universités d'Alsace

- Dr. Dr. Heinz-Peter Seidel, Generalkonsul der Bundesrepublik Deutschland in Straßburg

- Gérard Traband, Vice-Président du Conseil régional D’Alsace

- Dr. Veit Steinle, Leiter der Kunstabteilung im Ministerium für Wissenschaft, Forschung und Kunst Baden-Württemberg

Der vielfältige Austausch zwischen den beiden Bibliotheken soll durch die Partnerschaft ausgebaut werden. Dazu gehören insbesondere:

- Intensivierung des Erfahrungs- und Informationsaustauschs zwischen den beiden Bibliotheken

- Persönliche Kontakte (Kollegenbesuche, Fortbildungen)

- Gemeinsame Veranstaltungstätigkeiten (Ausstellungen, Vorträge)

- Gemeinsame Erschließung von Informationsressourcen

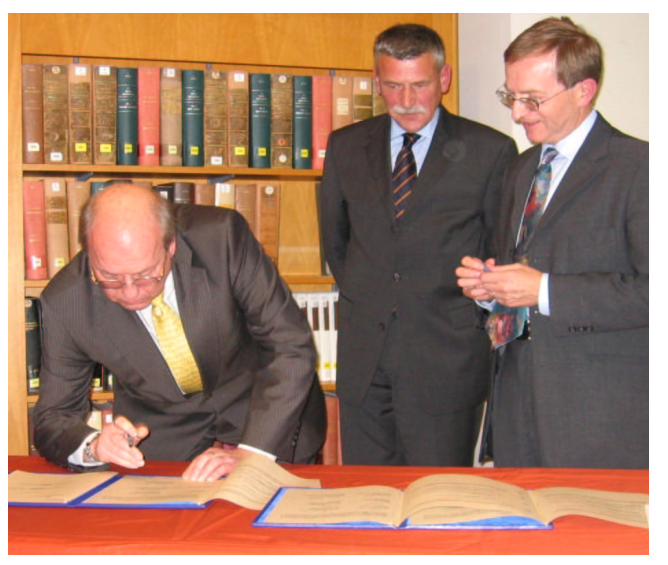

